reports that minor signs of liver damage can be detected in carriers, but there are certainly some who show no signs at all of liver disease. Carriers can be a dangerous source of infection with serum hepatitis, and Yvonne Cossart draws attention to the higher risk of antigenaemia in certain groups -for example, in children with Down's syndrome (mongolism) who live in institutions and in the normal populations of countries in the Far East, Africa, and Southern Europe. In these areas the carrier rate for Australia antigen is much higher than in the peoples of Nortern Europe and the United States. There is no evidence that people in warm climates suffer from a higher incidence of serum hepatitis than those in cool climates and the mode of virus transmission among them is unknown.

The discovery of Australia antigen was followed by reports of a different serum antigen, which appeared to be associated with infectious hepatitis. This antigen, the Milan or epidemichepatitis-associated antigen, was detected by precipitation reaction with an antiserum from a Milanese patient whose serum also contained antibody to Australia antigen. But A. J. Zuckerman presents evidence that this antigen is probably an abnormal serum lipoprotein which appears as the result of liver damage due to various causes and is not specifically associated with infectious hepatitis. In another article A. A. Ferris describes the discovery of an antigen in the faeces of patients with infectious hepatitis. The antigen cross-reacts with the larger Dane particles ${ }^{4}$ but not with the smaller particles of Australia antigen. Like Australia antigen it disappears during convalescence, but it has not so far been found in samples of serum. It appears to be relatively common in patients with infectious hepatitis and is rarely found in control groups, but its role in the disease requires further investigation.

Though serum hepatitis may occasionally be acquired by ingestion or inhalation, there is no doubt that parenteral inoculation or contamination of the skin by blood or blood products is the commonest route of transmission. Screening of blood for transfusion for the presence of Australia antigen is therefore of great importance, and W. d'A. Maycock discusses the control of the disease from the point of view of blood transfusion services. Fortunately the system of voluntary donation in Britain means that the incidence of Australia antigen among donors is very low. Nevertheless all blood for transfusion should nowadays be screened before use. The problem of serum hepatitis has been greatly increased by the formation in many hospitals of units for renal dialysis and transplantation. B. P. Marmion and R. W. Tonkin show that, in $1971,43 \%$ of these units reported cases of hepatitis among their patients. The mortality rate varies but is occasionally high. For example, in the severe outbreak in Edinburgh, $24 \%$ of the patients and $33 \%$ of the staff who contracted serum hepatitis died. Not surprisingly, the risk of infection is greatest to the members of staff who have the closest contact with the patients. In the Edinburgh outbreak it was found that the non-disposable connecting tube to the venous pressure gauge became contaminated with patients' blood and it was thought that this might have played a part in the spread of infection among the patients. Apart from obvious preventive measures, such as the careful avoidance of contamination with blood, patients on these units should be screened for Australia antigen at fortnightly intervals and the staff every three to six months. Marmion and Tonkin also give valuable advice on the control of infection in renal units, which includes methods for the cleaning and disinfection of those parts of the dialysis equipment which are non-disposable and which cannot be autoclaved.
1 British Medical Bulletin, 1972, 28, No. 2.

Blumberg, B. S., Gerstley, B. J. S., Hungerford, D. A., London, W. T., and Sutnick, A. I., Annals of Internal Medicine, 1967, 66, 924.

3 Le Bouvier, G. L., Fournal of Infectious Diseases, 1971, 123, 671.

4 Dane, D. S., Cameron, C. H., and Briggs, M., Lancet, 1970, 1, 695.

\section{Treating Incontinence Electrically}

B. R. Hopkinson ${ }^{1}$ has recently presented the results of five years' experience in treating incontinence of urine and rectal prolapse by electrical stimulation, using intra-anal electrodes. The conditions studied included rectal prolapse and incontinence in both adults and children, stress incontinence of urine in women, neurogenic incontinence of urine, incontinence of urine following prostatectomy and major pelvic surgery, and a triad of symptoms almost exclusive to women -namely, frequency, precipitancy, and urgency.

The fact that a simple device such as the anal plug electrode $^{2-4}$ might be used in the treatment and cure of such a wide range of distressing conditions should provoke a critical analysis of the present state of the electrical treatment of incontinence. K. P. S. Caldwell ${ }^{5-7}$ was the pio seer in the field of electrical control, using an implantable device devised at the Medical Research Council's Sphincter Research Unit in Exeter. The disappointing results of others, ${ }^{8}$ subsequently experienced by yet more workers, ${ }^{9} 10$ led to an increase in the popularity of the external electrode system devised by $S$. Alexander and D. Rowan ${ }^{11}$ for use in the female, in which a standard vaginal pessary served as an electrode carrier. Further similar devices have been designed. 1213

The present alternatives are either control by implantable apparatus, equally applicable to both sexes, or control by externally applied devices, also applicable to both sexes, except that the device used necessarily depends on the sex of the patient. The anal plug electrode is the only external electrical device available for use in adults of both sexes and children.

Much patient research undertaken in centres at London, Bristol, and Glasgow has not produced results as satisfactory as had been hoped. Reported success rates have fallen in consecutive publications, of which the latest ${ }^{14}$ shows success in just over $40 \%$ of adult women with stress incontinence. This is a lower figure than the $53 \%$ reported in a smaller group treated by Hopkinson, but the important difference between his series and any other published is that cures are claimed, whereas all others have reported control dependent only on the continued use of the prescribed apparatus.

Apart from the falling sucess rates, the other disturbing fact that has been reported is that there would not yet appear to be any objective method of predicting success in any one patient. This was one of the chief findings of L. Edwards, ${ }^{15}$ confirmed by others, ${ }^{16}$ and there would not appear to be any reason to doubt the validity of these observations. The inability to select patients for electronic treatment will constitute little in the way of a practical disadvantage if the use of external apparatus is considered, though it has to be remembered that each device costs up to $£ 50$, time may be lost, and the patient may suffer disappointment. With implanted electrodes the failure to predict success poses major problems. Should the procedure be limited only to those centres where it is undertaken frequently? Should it even be abandoned? There would appear to be little justification 
for its use in women, for in them the external apparatus, as well as lacking the risks of a major surgical procedure, does not seem to carry any lower degree of success.

The present state of the electrical control of incontinence is uncertain, to say the least. A recent statement ${ }^{17}$ that we should abandon continuous stimulation aimed at control rather than cure may present an extreme view. But there is much to be said for undertaking this form of treatment, particularly by the implant method, in specialist units. The techniques are not yet sufficiently understood nor in general sufficiently successful to be used outside such units.

${ }^{1}$ Hopkinson, B. R., Annals of the Royal College of Surgeons of England, 1972, 50,92 .

2 Hopkinson, B. R., and Lightwood, R., Lancet, 1966, 1, 297.

3 Hopkinson, B. R., and Lightwood, R., British fournal of Surgery, 1967, $54,802$.

4 Glen, E. S., Lancet, 1969, 2, 325.

5 Caldwell, K. P. S., Lancet, 1963, 2, 174.

Caldwell, K. P. S., Proceedings of the Royal Society of Medicine, 1965, 58,

' Caldwell, K. P. S., Annals of the Royal College of Surgeons of England, $1967,41,447$.

${ }^{8}$ Alexander, S., and Rowan, D., British fournal of Surgery, 1970, 57, 766. Riddle, P. R., Hill, D. W., and Wallace, D. M., British fournal of Urology, $1969,41,205$.

10 Edwards, L., Harrison, N., and Williams, J. P., British Medical fournal, 1971, 1, 543.

11 Alexander, S., and Rowan, D., Lancet, 1968, 1, 728.

12 Edwards, L., British fournal of Urology, 1971, 43, 211

13 de Soldenhoff, R., and McDonnell, H., British Medical fouranl, 1969, 4, 230.

14 Edwards, L. E., and Malvern, J., British fournal of Urology, in press.

15 Edwards, L. E., Hunterian Lecture, Royal College of Surgeons of England, 1971.

16 Glen, E. S., Unpublished transactions of European Continence Society Meeting, Exeter, 1971.

17 Collins, C. D., Proceedings of the Royal Society of Medicine, in press.

\section{Spiral Nerve Bands of Fontana}

One advantage arising from the study of medical history is that on occasion discoveries made by our distinguished forebears but subsequently forgotten or ignored prove to have unexpected significance even to modern medicine. Such is certainly true of the spiral nerve bands of Fontana, as described in a fascinating historical review by E. Clarke and J. G. Bearn. ${ }^{1}$ These authors point out that if the brachial plexus of a rat, for example, is exposed, white bands in the form of spirals can be seen on the nerve trunks with the naked eye, and a hand lens will show them clearly. They can also be seen in the peripheral nerves of many other animals as well as in man.

Few modern textbooks of zoology or human anatomy mention or illustrate this phenomenon. It was first clearly described by the Italian scientist, Felice Fontana, in $1781 .^{2}$ It is clear, as Clarke and Bearn point out, that other observers before Fontana had observed these bands but had not fully appreciated their significance, and Fontana was the first to suggest that they relate to the indispensible ability of peripheral nerves to tolerate stretching. To confirm the existence of the bands and to assess their significance Clarke and Bearn have done some "practical history." They have repeated the experiments of Fontana in order to evaluate more accurately his observations and interpretations, following the method of B. Zanobio. ${ }^{3}$ Studying the nerves of the brachial plexus, the femoral nerve, and the vagus, the lumbar and sacral spinal roots, and also lumbar muscle tendons in the rat, they used both modern microscopes and an old one, giving reasons for suggesting that the latter instrument was likely to be comparable to the one which Fontana employed. After killing the animals, the nerve trunks were exposed and examined with the naked eye, being illuminated first with an overhead operating lamp, then with vertical lighting from a Zeiss operating microscope, and thirdly with a spotlight at an angle of $45^{\circ}$ directed along the long axis of the nerve examined. Subsequently the nerve trunks were re-examined with an operating microscope at a magnification of $\times 6$, as used by Fontana, and photographed with bold vertical and oblique illumination. Small specimens of nerve were then separated by micro-dissection and mounted in physiological saline on slides for further study. Similar observations were made on specimens of human genito-femoral nerve excised 12 hours post mortem.

In the brachial plexus of the rat the spiral bands of Fontana can be seen with the naked eye under vertical lighting, but their appearance is much enhanced under oblique light. When gentle traction was applied to the nerve trunk in the intact animal, the spiral banding disappeared almost completely. With higher magnification under a modern miscroscope it was shown that the bands were due to nerve fibres pursuing an undulating or zigzag course. Similar appearances were observed in human nerves when divested of fascial coverings, but no such bands were found in lumbar and sacral spinal roots of the rat. Rat tendons also showed a banding finer than that of the nerve, again enhanced by oblique light and dispelled by stretching. The bands were less easy to see in the nerves of the rabbit and man, and the authors concluded that this was due to the larger amounts of connective tissue investing these nerves.

These interesting observations have confirmed Fontana's view that the single fibres of a peripheral nerve pursue a winding or zigzag course and that by means of an optical effect this produces an appearance of spiral banding seen on the fresh, unstained nerve at low magnification. When the nerve is gently stretched, the fibres straighten and the banding disappears. Little is yet known about the production and maintenance of this irregular or zigzag course of nerve fibres, even though it appears to be an essential morphological feature allowing the nerves to be stretched during movement without damage to, or disruption of, fibres. Yet, as Clarke and Bearn point out, this finding, so well described by an eighteenth-century investigator, relates to a vital characteristic of peripheral nerve.

\footnotetext{
1 Clarke, E., and Bearn, J. G., Brain, 1972, 95, 1.

2 Fontana, F., Traité sur le Vénin de la Vipère sur les Poisons Americains.

Florence, 1781.
${ }^{3}$ Zanobio, B., Physis, 1959, 1, 307.
}

\section{Sex and the Single Girl}

Teenagers tend to be both shy and egocentric, and many find it embarrasing to talk about their sexual activities with anyone other than their contemporaries. When this natural reticence is combined with less than ready availability of contraceptive advice and devices it is not surprising that a high proportion of girls' first sexual experiences should be unprotected against unwanted pregnancy. The report in this issue (p. 694) by Drs. C. McCance and D. J. Hall of the sexual behaviour of women students at Aberdeen University shows that a disturbingly large proportion seemed content to maintain a high level of sexual activity 